new training program was introduced: new nursing students now have to qualify in adult ICU as well as in pediatric ICU. The aim is to evaluate this new training program.

Methods Using a written questionnaire we asked students for their motivation and experience regarding this new training program. We kept records of which nurses took care of which patient in each unit.

Results

- A sufficient inflow of young graduates.

- Sufficient qualified nurses in PICU

- $50 \%$ of who initially wanted to graduate in adult ICU nursing only, became enthusiastic about working in PICU and now continue to work in PICU either fulltime or parttime.

- Nurses from the adult units can come and help in PICU which means closure of beds could be prevented.

- The possibility of admitting an ill child at an adult unit when there is no bed available at PICU.

- Which very likely leads to less refusals.

Conclusion A good cooperation between adult and pediatric ICU can be very beneficial for patient care. Particularly, training ICU nurses in adult AND pediatric ICU leads to better staffing in PICU.

\section{EVALUATING THE EXPERIENCE OF USING ONLINE BLENDED LEARNING DURING INDUCTION PROGRAMIME FOR NURSES IN A LEVEL 3 NEONATAL UNIT}

doi:10.1136/archdischild-2012-302724.1861

BS Mathew. Neonatal, Homerton University Hospital City University, London, UK

Background and Aims The Department of Health has published guidances to help the NHS improve the care provided for premature and sick babies. However, a report by BLISS, the baby charity highlighted a shortage of specialist neonatal nurses within the UK. This often results in recruitment of in-experienced workforce who lack necessary knowledge and skills to perform safely in their workplace. This paper presents qualitative findings from a study which evaluated blended learning approach used in an induction programme for such novice nurses. The aim of the study was to explore registered nurses' experience on the use of blended learning.

Methods The study employed a mixed method approach and collected data from 20 newly recruited nurses using purposive sampling. Data were collected using questionnaires and semi structured interviews. Data analysis was by thematic content analysis.

Results The analysis of data revealed that nurses had a positive and satisfactory experience of using online blended learning which has enabled them to adapt to the new environment quickly. Most of them did not have previous online learning experience but wanted this to be implemented in future education programmes. The major barriers faced by the nurses participating in online learning were lack of time and motivational factors.

Conclusion Using social networking as a learning tool has a great potential in supporting nurses to develop their knowledge and skills. Advance planning and preparation are essential to enhance the quality of their learning experience.

\section{IMPLEMENTATION OF THE DUTCH GUIDELINE FOR THE CARE OF EXTREME PRETERMS: "THE LEARNING CURVE"}

doi:10.1136/archdischild-2012-302724.1862

JM Wielenga, A Claassen, R van der Hoek, S Broekman. IC Neonatology, Women's and Children Clinic Academic Medical Center Amsterdam, Amsterdam, The Netherlands

Background and Aims The Dutch multidisciplinary evidence based guideline on treatment decisions of infants born with a gestational age of at least 24 weeks was released in autumn 2010. The implementation of this guideline on the NICU of the Women's and
Children Clinic/Academic Medical Center in Amsterdam the Netherlands was a complicated process.

Methods A multidisciplinary workgroup, 5 nurses, 5 neonatologists and a pharmacist composed a phased implementation procedure which consisted of the following activities:

A multidisciplinary meeting before and 10 months after implementation; Existing protocols were made evidence based; Working visits were made; A centre of expertise was established; A group of experienced nurses and doctors were steered as leaders in the care; Clinics and workshops for leading nurses; Clinics for all professionals concerned; Sessions for nurses to reflect and learn from each other; Evaluation of the implementation; and an Inventory to readjust existing guideline.

Results Due to media attention, an early start was forced. Protocols were not ready, material and equipment not available. The number of infants was higher than expected. The decision to perform the care for these infants by leading nurses resulted in agitation amongst other nurses. Their opinion was that complex nursing care should be performed by all experienced nurses. New rules resulted in a lack of clarity.

Conclusions Well-considered choices were made, however it appeared that nurses were in need of training on the job instead of planned theoretical training in advance.

\section{STUDYING THE HOSPITALIZATION CAUSES OF NEONATES FROM FARS PROVINCE AT THE NURSERY WARDS}

doi:10.1136/archdischild-2012-302724.1863

'M Edraki, ${ }^{2} \mathrm{H}$ Morravej, ${ }^{2 M}$ Rambod, ${ }^{2} \mathrm{~S}$ Montaseri. 'Shiraz University of Medical Sciences; ${ }^{2}$ Nursing and Midwifery, Shiraz University of Medical Sciences, Shiraz, Iran

Introduction and objective Immature neonates are exposed to be affected by Infectious, Pulmonary, Cardio-Vascular, Digestive, Metabolic and Endocrine diseases. The aim of present study is to survey the hospitalization causes of immature neonates at thenursery wards.

Materials and Methods This study is a descriptive research. The samples were consisting of 60 immature neonates less than 37 weeks and weight of below 2500 grams who had been hospitalized at the nursery wards of Shiraz city. These neonates were selected by simple sampling method.

Results The results of the study showed that, $53.5 \%$ of hospitalization causes of immature neonates at the hospital were affection to the Jaundice and Respiratory Distress Syndrome followed by Pneumonia which was the cause of $30.0 \%$ of cases. While, affection to the diseases like Sepsis, Hypoglycemia, Necrosing Enterocolitis, Metabolic disorders and Nosocomial infection were the cause of $8.3 \%, 5.0 \%, 3.3 \%, 1.7 \%$ and $1.7 \%$ of the hospitalization cases respectively. None of them were hospitalized due to the intra cerebral hemorrhage in this study.

Discussion and conclusion In this study, Jaundice, RDS and Pneumonia are among the most prevalent causes of hospitalization of immature neonates in the hospital.

It is recommended for the health care givers to have sufficient information in this regard and while facing immature neonates, pay more attention to their respiratory condition.

\section{EXPERIENCES OF PARENTS OF CHILDREN WITH CANCER: A BASIS FOR THE TRAINING OF NURSES}

doi:10.1136/archdischild-2012-302724.1864

Z Molazem, Z Yazdanpanahi, H Reisi. School of Nursing \& Midwifery Shiraz University of Medical Sciences and Health Services, Shiraz, Iran

Background and Aims Being confronted with the diagnosis of childhood cancer in the family causes various emotional reactions. 
This study aimed to determine the lived experiences of parents of children diagnosed with cancer in Iran.

Methods A qualitative approach was adopted using content analysis of semi-structured interviews carried out with 10 parents to capture their experiences of having children with cancer.

Results The following themes emerged from the analysis: being aghast by the diagnosis', 'living in the shadow of fear', 'uncertainty', 'struggle for taking control'.

Conclusions The findings of the study can provide nurses and other health professionals with a deeper understanding of Parents' view when they have a child suffering from cancer. It is important to health professionals involved in caring for these people. The study calls for more attention to parents of children with cancer and education to nurses.

\section{A WORK-TRAINING-UNIT ON THE NEONATAL INTENSIVE CARE UNIT (NICU). AN OPTIMAL LEARNIG CLIMATE?}

doi:10.1136/archdischild-2012-302724.1865

J Wielenga, M Hemmink, M Willemsen, C Bouwens, J Boltje. IC Neonatology, Women's and Children Clinic Academic Medical Center Amsterdam, Amsterdam, The Netherlands

Background and Aims On the NICU of the Women's and Children Clinic/Academic Medical Center in Amsterdam the Netherlands, newborns are transferred to regional hospitals for high dependency care. This had consequences for inexperienced nurses' preparation of their post graduate Intensive Care Neonatology training. These trainees had to work on the Intensive Care without the proper patients present. Therefore independency in caring was limited. To create a powerful and safe learning climate and to offer trainees the opportunity to become competent, a work-trainingunit was established.

Methods Preconditions were decided on: presence of an adequate amount of coaches, training focussing on situational coaching, four newborns, one coach and two pre-course trainees in the day or evening shift and preferably no trainees in the night shift and daily reflection and evaluation. Coaches were trained how to supervise and enhance the competency, expertise and independency of the trainees.

Results On the work-training-unit the trainees were offered the opportunity to care for post-IC and high dependency newborns, under constant supervision of their coach.

Trainees in the work-training-unit, achieved the acceptable level of functioning and usability sooner. An increase in performing nursing procedures and standard care in a uniform way was noticed. Trainees as well as coaches were extremely satisfied.

Conclusions The work-training-unit is a valuable addition to the learning possibilities of nurses as well as the organisation. It has positive side effects on work pressure, production and capacity but most of all it appears to be an optimal learning climate.

\section{LEVELS OF AWARENESS IN TEACHERS REGARDING CHILD NEGLECT AND ABUSE}

doi:10.1136/archdischild-2012-302724.1866

D Tugay. Statistical Department, Ministry of Health of Turkey, Health Resarch General Directorate, Ankara, Turkey

\footnotetext{
Aims In ourcountry, there are still no measurement tools having sufficient content and scope that aims at the awareness and any databank on this aspect, in spite of studies aimed at child neglect and abuse are maintained. With this study, it's aimed at evaluating awareness levels of teachers for child $\mathrm{n} \& \mathrm{a}$, and having gained the questionnaire in the Turkish literature.

Method In our study, "Child Abuse and Neglect Questionnaire for Teachers" that was developed byKerryann Walsh et al. has been used. The questionnaire has been applied to 1000 teachers who are
}

employed in 43 schools in the provincial centrum of Ordu, and has been completed with 400 teachers.

Results Teachers expressed that they have to report the suspicion of child n\&a in rate of $45.7 \%$ by National Education policies (Table1). A detection question of "What is the possibility of thischild being abused or neglected?" has been addressed to teachers for each of 32 stories. The average of given answers of the teachers aimed at the child n\&a in the stories is 3.01 over 5 , and is as "I am not sure". And the average of answers given related with reporting is 2.77 , and is probably between "no" and "I am not sure" (Table2). With the increase of determining the possibilities of n\&a, it's been seen that the reporting possibilities of teachers are increased (Table3).

Conclusions It could not be said that the awareness levels of teachers for child n\&a are sufficient. Therefore, the importance of education programmes for $\mathrm{n} \& \mathrm{a}$ in our country should be emphasised and a mandatory reporting procedure that is carefully systemised within the national education programmes should be conducted.

\section{EFFECT OF ALOEVERA GEL VERSUS LANOILIN OINTMENT ON NIPPLE SORE}

doi:10.1136/archdischild-2012-302724.1867

'M Gholami, ${ }^{2} \mathrm{R}$ Saeidi, ${ }^{2} \mathrm{M}$ Tafazoli. 'Neyshabur University of Medical Sciences; ${ }^{2}$ Mashhad University of Medical Sciences, Mashhad, Iran

Background and Aim A common, early postpartum concern of the breastfeeding mother is nipple pain Because of lack of effective treatment and Opponent results about lanolin, we decided to compare effect of lanolin ointment and Aloevera gel on nipple sore treatment.

Methods This clinical blind trial was performed in Mashhad, Iran from Feb 2008 to May 2009. Sampling was nonrandom and purpositive and then samples divided randomly into two groups.

Mothers were instructed to rub the preparation on their arms and if there wasn't cause allergy symptoms, rub it on the nipple after feeding the baby 3 times a day for 7 days and let to dry and don't wash it before next feeding. Researcher pretreatment, at 3rd and $7^{\text {th }}$ day filled Storr scale with interview and examination There was no any complications in mothers and infants because of drug usage.

Then data was analyzed with Spss 11.5 and Mann-witney, t student and t-paired tests.

Results A total of 100 samples enrolled with 50 samples in lanolin group and 50 samples in aloevera group.

nipple sore scoring decreased significantly at $3^{\text {rd }}$ day versus pretreatment $(\mathrm{p}=0.00)$ also at $7^{\text {th }}$ day versus pretreatment and $3^{\text {rd }}$ day $(\mathrm{p}=0.000)$.

nipple sore scoring decreased significantly at $3^{\text {rd }}$ day versus pretreatment $(p=0.00)$ also at $7^{\text {th }}$ day versus pretreatment and $3^{\text {rd }}$ day $(\mathrm{p}=0.000, \mathrm{p}=0.001)$.

Two groups were homogenous concerning at pretreatment day $(p=0.711)$, but there was a significant difference between 2 groups at $3^{\text {rd }}$ and $7^{\text {th }}$ day $(p=0.048, p=0.003)$.

Conclusion Aloe Vera is more effective than lanolin on nipple sore healing.

\section{PROBLEMS IN THE PROCESS OF WEANING IN BREAST-FED BABIES}

doi:10.1136/archdischild-2012-302724.1868

${ }^{1} \mathrm{~N}$ Çınar, 'D Köse, ${ }^{2 T}$ Nemut, 'S Altınkaynak. 'Department of Child Health Nursing; ${ }^{2}$ Sakarya University School of Health Sciences, Sakarya, Turkey

Background and Aim Sometimes weaning process may be problem for mothers and their babies. The study was done as descriptive 